\title{
Terminological dynamics in the context of the COVID-19 pandemic
}

\author{
[Dynamique terminologique dans le contexte de la pandémie de \\ Covid-19]
}

\author{
Ana-Marina Tomescu - Elena-Cristina Ilinca
}

DOI: 10.18355/XL.2021.14.04.09

\begin{abstract}
This article aims at presenting some peculiarities of the press discourse on the Covid19 pandemic, with an emphasis on the evolution of the specific terminology used in this context. It analyses written press articles which are informative texts, opinion texts, or science popularisation texts on the website of the Le Monde newspaper, which will be discussed from the point of view of the transmission of information and knowledge related to the evolution and management of the health crisis. As for the terminology studied in this paper, our analysis involves the identification and discussion of newly created terms, new usages of existing terms, or borrowings from other languages aimed at filling denominational gaps in French.
\end{abstract}

Key words: pandemic, terminology, terminography, translation

\section{Résumé}

Cet article vise à présenter quelques particularités du discours de presse lié à la pandémie de Covid 19, en mettant l'accent sur l'évolution de la terminologie spécifique utilisé dans ce contexte. Il s'agit de textes de presse écrite, de textes informatifs, d'opinion ou de vulgarisation scientifique parus sur le site Internet du journal Le Monde, qui seront analysés du point de vue de la transmission des informations et des connaissances liées à l'évolution et la gestion de la crise sanitaire. Quant à la terminologie analysée, il s'agit de l'identification et de la discussion de nouveaux termes créés, de nouveaux usages des termes déjà existants en français ou d'emprunts à d'autres langues visant à combler les lacunes dénominatives en français.

Mots-clés : pandémie, terminologie, terminographie, traduction

\section{Introduction}

La crise sanitaire de la Covid-19 à laquelle nous nous confrontons tous depuis plus d'une année a entraîné de nombreux changements à tous les niveaux de la société. Les nouvelles réalités liées à cette pandémie ont déterminé la création d'une terminologie spécifique afin d'assurer une gestion efficace de l'information, de satisfaire le " besoin de normalisation des communications et des échanges » (Roche, $2005: 57$ ) entre les autorités sanitaires, les responsables politiques et les citoyens.

Notre article se propose d'identifier et d'analyser des termes relatifs à la pandémie de coronavirus dans des textes de presse. Le point de départ de notre étude est représenté par l'allocution liminaire du directeur général de l'Organisation Mondiale de la Santé, Tedros Adhanom Ghebreyesus, sur la Covid 2019, présentée le 11 mars 2020. Suite à une évaluation 24 heures sur 24, l'OMS a décrit brièvement le contexte sanitaire. Les données chiffrées et le besoin de répondre aux nouveaux défis dans le domaine de la santé ont mené l'OMS à qualifier la Covid-19 de première pandémie provoquée par un coronavirus, à cause de sa gravité (plus de 118000 cas dans 114 pays et 4291 personnes avaient perdu leur vie à ce moment-là), les niveaux alarmants de propagation (le nombre de cas en Chine s'était multiplié par 13) et d'inactivité (à cause du manque de capacités, de détermination ou de ressources).

XLinguae, Volume 14 Issue 4, October 2021, ISSN 1337-8384, eISSN 2453-711X 
Dans notre analyse nous allons faire référence aussi à plus d'une dizaine d'articles parus depuis mars 2020 jusqu'au mai 2021 sur le site Internet du journal Le Monde. Il ne s'agit pas d'une communication spécialisée faite par des spécialistes, il s'agit d'articles informatifs ou d'opinion, d'articles de vulgarisation scientifique, mais les connaissances présentées sont acquises par l'intermédiaire des spécialistes. Nous avons choisi de travailler sur un corpus de presse en considérant que les mass media ont été le principal vecteur de communication pendant cette période de crise sanitaire.

\section{Quelques précisions étymologiques}

Après la déclaration de l'Organisation Mondiale de la Santé mentionnée plus haut, le terme pandémie a fait florès dans la presse écrite de tous les coins du monde et surtout sur Internet, même s'il " n'est pas un mot à utiliser à la légère ou avec légèreté ", selon les propos du directeur de l'OMS. Depuis plus d'un an, le terme a fait son chemin dans nos esprits et il est entré peu à peu dans le langage courant.

Quelques événements qui semblent avoir contribué à forger le sens du mot sont liés à l'émergence de fléaux infectieux majeurs, tels que la grippe (la grippe russe de 1889, la grippe espagnole de 1918), la peste noire (au XIV ${ }^{\text {ème }}$ siècle), le choléra (en 18311832), la dengue, le sida. Du point de vue étymologique, le mot vient du grec ancien $\pi \alpha v \delta \eta \mu i ́ \alpha$, pandêmía (« le peuple tout entier »), composé de $\pi \tilde{\alpha} v$, pân («tout ») et $\delta \tilde{\eta} \mu \circ \varsigma$, dêmos («peuple»). Le premier ouvrage lexicographique français qui a attesté ce mot a été le Dictionnaire universel français et latin (appelé aussi Dictionnaire de Trévoux), dans son édition de 1752. Il s'agissait d'un emprunt à l'anglais (pandemic), utilisé en 1666 par le médecin britannique Gideon Harvey, dans son livre sur la tuberculose. Le dictionnaire informatisé Trésor de la langue française définit la pandémie comme une " épidémie qui affecte une partie significative des populations sur une région importante du globe, plusieurs pays voire plusieurs continents ", ce qui correspondait déjà à la réalité sanitaire présentée dans la déclaration du mois de mars 2020 : maladie infectieuse, vaste extension géographique, propagation exponentielle et inattendue par le biais d'une transmission. Mais, l'OMS attirait l'attention sur le caractère de nouveauté de cette pandémie, puisque « nous n'avons jamais vu auparavant une pandémie déclenchée par un coronavirus. »

\section{Caractéristiques linguistiques des textes de presse liés à la pandémie}

Les textes que nous avons inclus dans notre corpus ont comme objectif la communication formelle, parfois institutionnalisée. Les textes relatifs à la pandémie, publiés dans le journal Le monde depuis mars 2020 jusqu'au mai 2021, ont comme but d'informer, de décrire, de faire comprendre la situation sanitaire en question. Parfois, les auteurs essaient de convaincre leurs lecteurs et ils recourent à des procédés multiples : explication, justification, comparaison, emploi des citations d'opinions ou d'idées de certains spécialistes. Il s'agit d'un « discours préparé, rédigé, qui déroule un fil narratif, descriptif, voire argumentatif pensé en progression, duquel redondance et retour correctif (par exemple) sont exclus. Cela signifie que le terme apparait une fois (soit en dénomination, soit en définition) à un moment précis car répondant à un besoin communicationnel, mais qu'il ne fait pas l'objet d'une deuxième mention pour une éventuelle redéfinition, par exemple. " (Vargas, 2009 : 15). Prenons un exemple :

"Si "l'entraînement ne garantit pas une récupération totale de l'odorat chez $100 \%$ des personnes, il multiplie par deux ou trois les chances", souligne Moustafa Bensafi, spécialiste de l'odorat. "Il est d'autant plus efficace qu'il est long". » (lemonde.fr, le 16 avril 2020) 


\subsection{Types de phrases}

L'emploi de la phrase déclarative est prédominant. Ce type de phrase aide à :

- décrire une situation, un phénomène (par exemple le fonctionnement des vaccins) :

" Tous les vaccins ont le même but : apprendre au système immunitaire de l'organisme à reconnaître et à fabriquer ses défenses - les anticorps spécifiquement contre le Sars-CoV-2. Ces défenses pourront ainsi neutraliser rapidement le virus lorsqu'elles le croiseront. » (lemonde.fr, le 30 décembre 2020)

- introduire des statistiques, des données chiffrées :

" Le nombre de patients Covid-19 à l'hôpital a lui aussi continué à reculer, à 28 930, contre 29487 la veille. Au cours des dernières vingt-quatre heures, 271 malades du Covid-19 en sont morts à l'hôpital. » (lemonde.fr, le 30 avril 2020)

- établir un rapport de cause à effet (entre la pandémie et la santé des Français les derniers mois) :

«Un rapport portant sur 4 milliards de prescriptions entre mars 2020 et avril 2021 confirme la dégradation de la santé mentale des Français sous l'effet de la crise sanitaire. » (lemonde.fr, le 27 mai 2020)

En ce qui concerne l'emploi de la phrase interrogative, il y a deux situations où nous rencontrons ce type de phrase : dans le titre de l'article ou dans la formulation d'une question, principale ou secondaire, à laquelle l'auteur de l'article journalistique ou le scientifique tente de répondre :

«Covid-19 et perte de goût et d'odorat durable : quelle rééducation?» (lemonde.fr, le 16 avril 2020)

«Les Français seront-ils assez nombreux à accepter de se faire vacciner pour atteindre l'immunité collective, et ainsi empêcher une quatrième vague ? » (lemonde.fr, le 30 mai 2020)

- exposer une polémique, une thèse adverse :

« Le débat a surgi dès l'annonce par Pfizer et BioNTech de résultats intermédiaires très positifs quant à l'efficacité de leur vaccin, lundi 9 novembre. » (lemonde.fr, le 17 novembre 2020)

\subsection{Tendance à la dépersonnalisation, par :}

- la prédominance de la $\mathrm{III}^{\mathrm{e}}$ personne, singulier et pluriel:

« ils ont réussi à maîtriser rapidement la circulation du virus », « ils subissent un rebond épidémique », « elle produit des vaccins », etc. (lemonde.fr, le 29 mai 2020)

- l'absence de la $\mathrm{I}^{\text {ère }}$ singulier et de la IIe personne, singulier et pluriel. 
- l'emploi des expressions impersonnelles :

"Faut-il rendre la vaccination contre le Covid-19 obligatoire? " (lemonde.fr, le 17 novembre 2020)

«... il est prématuré d'imaginer l'étendre à l'ensemble de la population à ses débuts. » (lemonde.fr, le 17 novembre 2020)

"Il est tentant de conclure que cette maladie ne serait pas si dangereuse. " (lemonde.fr, le 14 mars 2020)

" Mais il est clair que d'autres virus tuent une proportion bien plus élevée des personnes infectées. » (lemonde.fr, le 14 mars 2020)

"Il est nécessaire de disposer d'un échantillon plus large pour mieux étudier ces séquelles et pour mesurer le taux d'anticorps pour le SARS-CoV-2.» (lemonde.fr, le 9 janvier 2020)

- l'emploi des tournures passives, avec omission de complément introduit par la préposition par:

« Ces chiffres, qui reposent sur les bilans quotidiens des autorités nationales de santé, sont globalement sous-évalués. » ; "Les bilans officiels avancés ... 》 (par les autorités) ; "ont révélé la présence du variant B.1.617.2 du Covid-19 sur des prélèvements effectués sur des membres d'équipage du Shandong-Da-Zhi » (par des personnes autorisées) (lemonde.fr, le 21 mai 2020)

Ces caractéristiques, propres aux langues de spécialités, ont été observées aussi par d'autres spécialistes qui mettent en évidence les procédés de distanciation : « Les langues de spécialité(s) se caractériseraient par une syntaxe réduite et un vocabulaire spécialisé. Il s'agirait d'un sous-système moins complet de la langue générale qui privilégie certaines tournures ou constructions, certains modèles : ex. la nominalisation dans la presse, les procédés de distanciation (ex. passivation, locutions impersonnelles) dans le discours scientifique, juridique, etc. »(Binon, Verlinde, $2002: 40)$.

2.3. «Perspective atemporelle », comme le souligne G. Vigner et A. Martin (1976 : 32) et plus tard R. Kocourek (1982 : 51), par l'emploi fréquent du présent de l'indicatif, un temps verbal qui sert à situer les faits au moment de l'énonciation. Le même auteur soulignait le fait que « c'est le présent qui prédomine dans des écrits scientifiques ( $85 \%$ de toutes les formes verbales), $10 \%$ des occurrences étant réservées au passé composé et au futur ; le reste est réparti entre les autres temps et modes. »

" "La caractéristique principale de ce virus est qu'il se transmet rapidement dans l'air. La concentration de virus dans la gorge et la salive augmente rapidement, et il se répand très vite dans l'environnement proche", a déclaré le ministre de la santé, Nguyen Thanh Long, lors d'une réunion nationale sur la pandémie. » (lemonde.fr., le 30 mai 2021)

«Les résultats de l'étude, réalisée avec des hôpitaux universitaires français, montrent qu'une dose du vaccin AstraZeneca "fonctionne très peu contre les variants indiens et sud-africains ». Une seule dose de ce vaccin apparaît donc «peu ou pas du tout efficace " contre le variant indien, souligne le chercheur. » (lemonde.fr, le 29 mai 2021) 


\subsection{Préoccupation constante pour concision et précision}

- emploi des acronymes. L'idée la P. Lerat (1995) est soutenue aussi par d'autres linguistes. J. Percebois souligne que « depuis une cinquantaine d'années s'observe un recours croissant aux sigles et aux acronymes, dans tous les milieux et dans tous les types de discours, à l'écrit comme à l'oral » (2001 : 627). En revanche, il y a des spécialistes qui considèrent qu'il y a trop de sigles et d'acronymes empruntés à l'anglais dans les textes médicaux francophones (Jammal, $1992: 213$ ).

« S'ils subsistent au-delà de deux mois, il est recommandé au généraliste d'orienter vers un spécialiste $O R L$, voire de réaliser une IRM. » (lemonde.fr, le 16 avril 2020)

«Un test diagnostique $R T$-PCR de détection sur des prélèvements nasopharyngés revient positif pour le $S A R S$ - $C o V$-2. » (lemonde.fr, le 7 avril 2020)

Le terme Covid ou covid représente lui-même l'acronyme de corona virus disease. Dans notre corpus, on peut remarquer l'usage alternatif du terme au masculin (le covid 19) et au féminin (la covid 19). Cette hésitation peut être expliquée par la confusion entre la dénomination du virus (SARS-CoV-2, masculin) et celle de la maladie (COVID-19, féminin). En mai 2020, l'Académie française se prononce sur son site Internet pour l'usage au féminin en précisant que les sigles et acronymes ont le genre du nom qui constitue le noyau du syntagme dont ils sont une abréviation. En vertu de cette règle, covid 19 est de féminin, car le mot de base est le mot maladie. On peut constater toutefois la préférence des usagers pour la forme au masculin, d'où l'enregistrement des deux formes dans certains dictionnaires ( $L e$ Petit Robert en ligne, par exemples).

\section{Emploi des termes à étymologie grecque et latine}

«La maîtrise du vocabulaire médical repose sur la connaissance des racines grecques et latines » (Rouleau, 1995 : 32). Pour s'exprimer de manière concise et correcte, pour se familiariser avec les termes médicaux, il vaut mieux de connaître l'étymologie des termes employés. Dans les exemples que nous avons mentionnés antérieurement, nous avons vu que le langage de la pandémie est composé de termes scientifiques empruntés au grec et au latin mais aussi d'expressions imagées empruntées à la vie courante. Nous avons rencontré :

- des préfixes tirés du grec:

a- (= privé de): asymptomatique, anosmie, agueusie, atypique;

anti- (contre): anticorps, antigène;

broncho- (gorge): broncho-pneumonie;

cyano- (bleu): cyanose;

dys- (avec difficulté): dyspnée, dyspepsie, dysfonction;

épi- (sur): épidémie;

hémo- (sang): hémorragie, hémoptysie;

oxy- (acide, aigu): oxygène, oxygénothérapie;

pan- (tout): pandémie, pandémique;

rhino- (nez): rhinopharyngite;

- des suffixes tirés du grec:

-algie (douleur): myalgie, névralgie;

-drome (course): vaccinodrome, syndrome;

-phagie (action de manger): odinophagie;

-phobe (ennemi de): vaccinophobe;

-phile (ami de): vaccinophile;

XLinguae, Volume 14 Issue 4, October 2021, ISSN 1337-8384, eISSN 2453-711X 
- des préfixes tirés du latin:

im- (dans): immunité, immunisation, immunogène;

in- (dans): incidence, incubation, infecter, infection, insufficience respiratoire,

injection de rappel, intubation, intuber;

inter- (entre): interruption, intervention;

pré-(devant): précaution, présymptomatique;

pro- (en avant): propagation, protéine de spicule;

re-(de nouveau): reconfiner, reconfinement;

super (au-dessus): supercontaminateur, superconamination;

trans- (au delà de, à travers): transmission;

\section{Création d'un nouveau vocabulaire}

La crise sanitaire de 2019 a modifié notre façon de parler et a investi notre langage. Nous avons tous vu émerger de nouveaux mots, qui pourraient être classifiés en plusieurs catégories : mots savants existants, mais inconnus au public large et qui étaient utilisés seulement par les spécialistes, mots qui ont changé de sens et de nouveaux mots inventés pour décrire la réalité sanitaire actuelle, complètement inédite. Les professionnels du domaine médical se retrouvent dans la même situation, car « le spécialiste ne trouvant pas toujours dans la langue générale tous les éléments dont il a besoin pour exercer sa profession, il a dû créer de nouveaux termes. » (Rouleau, 1995 : 30).

\subsection{Le virus, la confirmation de la pandémie, la transmission}

Depuis plus d'un an, les autorités parlent d'un pic de la première/deuxième vague de la pandémie. En fonction de l'évolution de la pandémie (recul, stabilisation, reprise), les autorités ont essayé d'aplatir la courbe, c'est-à-dire à faire baisser le taux de reproduction du virus. Par exemple, dans les centres de soins de longue durée ou dans les maisons de retraite, la mortalité a été très élevée.

«Les personnes âgées sont les premières victimes de l'épidémie. » (lemonde.fr, le 15 avril 2021)

Nous voudrions accorder une attention spéciale au mot épicentre, utilisé dans des contextes tels que :

"Coronavirus : comment l'Europe est devenue l' "épicentre" de la pandémie. » (lemonde.fr, le 18 mars 2020)

Dans la phrase ci-dessus, le mot épicentre a acquis un nouveau sens (le continent où le virus se manifeste d'une manière intense, l'Europe est devenue donc le nouveau foyer de la pandémie, après la Chine), tout à fait différent de son sens premier, mentionné par les dictionnaires : point de la surface terrestre où un séisme est le plus intense.

Depuis le début de la pandémie, les théories du complot ne cessent de déferler partout dans le monde (pangolin, chauve-souris, laboratoires chinois, contamination par produits surgélés). Dans ce cas on parle d'infodémie, c'est-à-dire « désinformation ou malinformation au sujet d'une épidémie ", un mot présent dans les dictionnaires depuis 2003, après l'épidémie de SRAS. En outre, la propagation du variant indien/britannique a semé la confusion chez les scientifiques et, dans certaines régions ce phénomène a contribué à l'explosion de la pandémie. 


\subsection{Formes cliniques, symptômes et diagnostics}

Dès le début de la pandémie, les spécialistes ont essayé de décrire les divers symptômes de la Covid-19, étant donné le fait que les coronavirus appartiennent à une catégorie de virus qui provoquent des maladies allant d'un simple rhume à des pathologies plus sévères, neurologiques, parfois mortelles. Les signes cliniques varient d'une personne à l'autre : fièvre, faiblesses musculaires, maux de tête, toux sèche, frissons, fatigue exagérée, essoufflement, troubles digestifs, nausée, neurones olfactifs endommagés, dysfonctionnements rénaux, etc.

« Alors que la plupart des patients atteints de Covid-19 développent de la fièvre, une toux, de la fatigue, une gêne respiratoire, il apparaît que chez certains patients, la maladie peut donc se présenter sous une forme neurologique. " (lemonde.fr, le 7 avril 2020)

Pour parler de la perte du goût et de l'odorat, certains auteurs utilisent les termes scientifiques, anosmie et agueusie. En cas de persistance durable, à quinze jours, de ce type de symptôme, le traitement et même la rééducation s'imposent :

« Mettre en route le plus rapidement la rééducation est primordial car cela reste pour le moment le seul traitement ayant prouvé son efficacité lors de prise en charge d'anosmie post-virale » (lemonde.fr, le 16 avril 2020)

Même en cas d'absence de symptômes, le porteur sain, appelé aussi asymptomatique, est contagieux. Il s'agissait de formes cliniques encore peu connues et les premières descriptions cliniques de l'infection à ce type de coronavirus attiraient l'attention sur l'existence de quelques cas bien graves :

« un cas d'encéphalopathie hémorragique aiguë nécrosante chez une femme testée positive pour le SARS-CoV-2 » (lemonde.fr, le 7 avril 2020)

\subsection{Hôpital, protection, tests de dépistage et vaccins}

Dans la lutte contre la pandémie, les autorités ont imposé le port du masque chirurgical jetable ou des visières pour le personnel médical. Une autre mesure était celle de recourir aux tests de dépistage de la covid 19, à partir de prélèvements salivaires ou à l'écouvillonnage du fond de narine.

« le masque doit couvrir nez et menton sans couture sagittale (sur le nez, dans le sens de la hauteur) » (lemonde.fr, le 28 janvier 2021)

«On ne peut qu'être frappé en constatant à quel point la question du masque est au cœur de tous les discours sur la pandémie et son traitement. » (Bacot, 2020). Il s'agit d'un mot dont l'étymologie n'est pas trop claire, et dont le premier sens désignait un objet utilisé pour déguiser ou dissimuler l'identité d'une personne. Dans le domaine médical, il s'agit d'un appareil qui permet de faire inhaler de l'oxygène tandis que dans le domaine militaire c'est l'appareil qui assure la protection contre les diverses radiations (masque à gaz). En contexte pandémique, le masque chirurgical représente l'étoffe qui offre la protection contre l'infection au coronavirus.

«Ou que la frontière entre des gouttelettes plongeant vers le sol et des aérosols plus volatils serait une taille de cinq micromètres? » (lemonde.fr, le 27 mai 2021) 
Nous avons remarqué l'emploi fréquent des mots construits à l'aide de la dérivation : l'écouvillonnage - l'écouvillon ; la salive - salivaire, thrombose - thrombotique thrombopénie, etc.

Malgré les soins intensifs dans les zones chaudes des hôpitaux submergés, dans les services de réanimation, le nombre des infectés a augmenté. Pour sauver la vie des patients, les médecins et les infirmières ont recours à la ventilation (appelée aussi oxygénothérapie), soit non-invasive soit invasive (appelée aussi intubation).

De l'autre côté, les autorités ont eu des difficultés dans la mise en place des stratégies de vaccination.

"Cette étude révèle la survenue fréquente de caillots sanguins dans les veines profondes et dans la circulation sanguine pulmonaire, autrement dit une fréquence élevée de maladie thromboembolique veineuse chez les patients Covid19. » (lemonde.fr, le 11 mai 2020)

L'adjectif veineuse est appelé par Rouleau adjectif construit en hypallage, parce qu' " il y a hypallage lorsqu'est attribué à un mot ce qui, dans les faits, convient à un autre mot qui, lui, est absent. » (1995 : 37), car l'adjectif se rapporte au système des veines.

\subsection{Mesures gouvernementales}

Pour traverser la crise sanitaire et garder la santé publique des Français, le président Emmanuel Macron a déclaré « la guerre » contre la COVID-19 et a pris plusieurs mesures sanitaires. Comme il s'agissait d'un état d'urgence sanitaire, chacun devait rester chez soi/rester à la maison, respecter les gestes barrière et garder la distanciation sociale/physique. Il faudrait éviter tout voyage non-essentiel.

L'interdiction de se déplacer sur le territoire du pays a été vulgarisée dans la presse écrite et audio-visuelle par plusieurs expressions, telles : confinement national, confinement partiel, confinement spontané. Dans une émission diffusée à la FranceInfo, le 4 mai 2021, le professeur de linguistique et conseiller scientifique du Petit Larousse, Bernard Cerquiglini, a parlé du changement de sens du mot confinement. Au début, il était utilisé dans le domaine juridique (isolement des brigands), ensuite il est passé dans le domaine de l'énergie nucléaire (précaution prise pour empêcher la dissémination des produits radioactifs). Les derniers mois, il a pris le sens que nous connaissons tous aujourd'hui (mesure sanitaire de restriction) et c'est cet emploi qui est courant actuellement. Sandrine Reboul-Touré, spécialiste du discours, a expliqué la préférence pour l'emploi de ce mot, qui n'affole pas les gens. Son synonyme, enfermement, nous aurait fait penser à l'idée de folie, d'asile. Par dérivation, confinement a produit encore le confiné, le déconfinement, le reconfinement.

Quant au mot quarantaine, nous pouvons remarquer de nouveau un changement de sens. Au début la quarantaine visait une durée de 40 jours, si quelqu'un était de retour d'une zone d'épidémie vers une zone non atteinte. À présent, suite aux mesures sanitaires à l'international, la quarantaine est censée à une période de 14 jours, ce qui correspond à l'incubation de certaines maladies infectieuses.

Face à une situation épidémique "maîtrisée mais fragile», Jean Castex a annoncé, jeudi 14 janvier, l'avancée du couvre-feu de 20 heures à 18 heures sur l'ensemble du territoire national. (lemonde.fr, le 15 janvier 2021)

En ce qui concerne le mot couvre-feu, le sens initial faisait référence à la sonnerie qui donnait le signal d'éteindre les lumières et de ne plus quitter le domicile. Le sens actuel a aussi un aspect temporel : sortir avant/après le couvre-feu. 
La création de nouveaux termes est fondée sur un travail important de conceptualisation afin de trouver une dénomination univoque, c'est-à-dire « un moyen d'expression qui permette à la fois de prévenir les erreurs d'interprétation et d'empêcher les fautes de raisonnement » (Frege, apud Roche, 2007 : 5). Un terme qui a suscité de nombreux débats en France c'est le terme de distanciation sociale, une traduction de social distancing, qui a commencé à être remplacé dans les discours publiques par distanciation physique, suite aux explications des linguistes: distanciation sociale (Dumazedier, Ripert, 1966) est un terme utilisé plutôt dans le domaine de la sociologie afin de désigner la distanciation entre les classes sociales, en d'autres mots le refus de se mêler à d'autres classes sociales. En conséquence, en raison de ses connotations politiques et culturelle, le terme distanciation sociale est à éviter dans le contexte de la pandémie.

\subsection{Secteurs touchés}

Dans son allocution liminaire, le directeur général T. A. Ghebreyesus précisait qu'il ne s'agissait pas seulement d'une crise sanitaire, mais d'une crise qui touchera tous les secteurs. Les mesures prises par les autorités et le changement des pratiques et de la routine de tous les jours ont entraîné l'apparition de nouveaux termes ou l'usage intensif de certains termes déjà existants dans la langue : télétravail, coronapiste, distanciel, tout-distanciel, enseignement à distance, enseignement virtuel.

«Rappelant que le télétravail "peut être considéré comme une des mesures les plus efficaces pour prévenir le risque d'infection au SARS-CoV-2", le nouveau protocole stipule que "les employeurs fixent dans le cadre du dialogue social de proximité, un nombre minimal de jours de télétravail par semaine, pour les activités qui le permettent". » (lemonde.fr, le 26 mai 2021)

«La volonté est là mais, en pratique, le gouvernement ne peut pas imposer aux élus locaux de modifier l'usage d'une chaussée dont ils ont la responsabilité, comme c'est le cas avenue du Prado à Marseille, où la métropole Aix-Marseille a fait supprimer une "coronapiste", en début de semaine. » (lemonde.fr, le 30 mai 2020)

«Malgré le distanciel, pas de décrochage massif pour les étudiants de première année » (lemonde.fr, le 16 mars 2021)

«Il est encore trop tôt pour le dire car c'est arrivé précipitamment, mais nous avons pu réfléchir aux difficultés que l'on pourrait rencontrer si l'on devait passer au tout-distanciel. Sur les plans pédagogique et relationnel, l'enseignement à distance ne permet pas la même approche des étudiants. [...] Tout est-il à rejeter dans l'enseignement virtuel ? (lemonde.fr, le 18 juin 2020)

\subsection{Emprunts anglais}

On peut constater aussi toute une série d'anglicismes utilisés dans l'espace francophone et pas seulement. Certains d'entre eux ont été remplacés dans les discours publics par leur traduction française ou remplacés peu à peu par des termes déjà existants dans la langue. À titre d'exemple : cluster ou foyer de contamination, lockdown, qui a été vite remplacé par le terme confinement, social distancing, au début traduit par distanciation sociale, remplacé par distanciation physique, contacttracing/tracking pour désigner l'activité de pistage, de traçage des cas-contacts, impacter, au lieu d'influencer, avoir des conséquences, click and collect, corona bond. Les guillemets sont souvent utilisés afin de délimiter les emprunts du reste de la phrase :

XLinguae, Volume 14 Issue 4, October 2021, ISSN 1337-8384, eISSN 2453-711X 
«Cela fait plusieurs mois qu'ils clament leur peur que la zone d'attente pour passagers en instance (ZAPI) de l'aéroport Roissy-Charles-de-Gaulle ne se transforme en cluster, sans avoir l'impression d'être entendus. » (lemonde.fr, le 28 avril 2021)

"Vous travaillez au sein d'une brigade de "contact tracing" ? " Racontez-nous (lemonde.fr, le 11 septembre 2020)

«Pour Claude Onesta, le report des Jeux olympiques "va impacter toute l'organisation du sport en France “» (lemonde.fr, le 24 mars 2020)

"Phénomène nouveau, les libraires qui n'ont pas de site pratiquent du "click and collect" artisanal : les clients téléphonent ou envoient un message sur les réseaux sociaux pour réserver un ouvrage et fixer un rendez-vous pour venir le chercher. » (lemonde.fr, le 5 novembre 2020)

« Reste la dernière étape, le lancement d'un grand emprunt européen spécifique, un "corona bond ", comme le suggère l'Italie. » (lemonde.fr, le 20 mars 2020)

Ces situations de variation dénominative ou terminologique peuvent être expliquées par le fait que la communication au niveau mondial se fait essentiellement en anglais, les autres langues ont besoin de temps afin de trouver la meilleure solution de dénomination, la solution la plus rapide étant l'emprunt direct à l'anglais.

\section{Retombées de la pandémie sur le travail terminologique et la traduction}

La crise sanitaire de 2020-2021 causée par le coronavirus a affecté tous les niveaux de la société, elle a déterminé des changements au niveau des pratiques dans tous les domaines d'activité. Tout le monde s'est retrouvé devant une quantité énorme d'information spécialisée, la plupart du temps vulgarisée afin d'atteindre toutes les couches sociales. Au niveau du travail terminologique et terminographique, les spécialistes ont pu observer une forte dynamique terminologique qui reflète ces changements profonds au niveau de la société : l'utilisation des emprunts aux autres langues (surtout anglais), la création de nouveaux termes, le glissement de sens déterminé par un nouvel usage dans le cas de termes déjà existants.

On peut constater l'élaboration de nombreux glossaires et lexiques relatifs à la pandémie ou l'enrichissement des bases de données terminologiques et des dictionnaires avec de nouvelles entrées. La création de ces produits terminologiques dépend de la qualité et la richesse des ressources linguistiques majoritairement disponibles maintenant en ligne mais dans des formats dispersés et hétérogènes, ce qui rend parfois difficile leur traitement automatique (Roche et al, 2019).

Les éditions Larousse ont annoncé l'inclusion dans Le petit Larousse 2022 un nombre record de cent soixante-dix nouveaux termes, la plupart liés à la pandémie de covid19: patient zéro, quatorzaine, cluster, coronapiste, nasopharyngé ne sont que quelques exemples.

Le petit Robert 2022 propose aussi des termes liés à la crise sanitaire. Prenons quelques exemples : corona, virus aéroporté, cas-contact, syndrome de détresse respiratoire aigüe, vaccinodrome, couverture vaccinale.

L'évolution de la pandémie a déterminé un échange d'information extrêmement dynamique d'un pays à autre, d'une langue à autre. La traduction a eu un rôle essentiel dans ce transfert transnational de recherche, connaissances, mesures adoptés, pratiques en vue de lutter contre ce virus. L'industrie de la traduction a connu une forte augmentation de demandes de traduction dans le domaine de la santé, sciences de la vie, pharmaceutique. Devant traduire de termes complètement nouveaux, le traducteur s'est retrouvé dans la situation de faire du travail terminologique en essayant de trouver les bonnes équivalences interlinguistiques ou intralinguistiques ou de créer lui-même des termes ad hoc dans la langue cible afin de combler les lacunes dénominatives relatives aux nouvelles réalités. 


\section{Conclusions}

$\mathrm{Au}$ terme de cette étude, on peut dire que l'évolution de la société dans le contexte pandémique des dernières deux années a déterminé une dynamique inattendue au niveau du langage, des stratégies de communication et de travail. Quant aux médias, ils ont dû transmettre une quantité immense d'information de la part des responsables politiques ou sanitaires : résultats des études et des enquêtes, nouvelles mesures à prendre, nouvelles règles à respecter, changement des conditions de travail, études, voyages, etc.

L'abondance des échanges communicationnels liés à l'apparition et à la propagation du coronavirus a mené à l'usage simultané d'une nouvelle terminologie dans toutes les langues, terminologie que presque tous les humains ont dû connaître et comprendre afin de s'adapter aux nouvelles conditions de vie. Dans ce nouveau contexte, la langue française a démontré une grande capacité d'adaptation par la proposition de nouveaux termes afin de combler des lacunes dénominatives temporaires et d'éviter l'utilisation de trop d'anglicismes. Nous avons pu identifier dans notre étude de nouveaux termes créés par dérivation, par composition, par télescopage, des termes qui ont reçu de nouveaux sens, des emprunts à l'anglais capables d'exprimer des idées de façon plus synthétique. Reste à voir quelle sera la durée de vie de ces termes issus dans de telles circonstances. Nous avons pu voir aussi des cas où la variation dénominative peut être une source d'incertitude, de confusion ou de malentendu pour le terminologue, le traducteur, mais aussi pour le destinataire final, le citoyen.

Enfin, il faut souligner ici une fois de plus le rôle essentiel des institutions de normalisation de la langue qui doivent intervenir afin d'établir des normes de langue pour une communication précise, efficace, transparente et éthique au niveau officiel, institutionnel et sociétal.

\section{Bibliographic references}

BACOT. P. 2020. Masque. In : Société d'étude des langages du politique. Available online: https://selp.eu/figures/masque/, consulté le 15 mai 2021.

BINON, J. - VERLINDE, S. 2002. Les langue (s) de spécialité (s) : mythe ou réalité ? Lexicographie et langue (s) de spécialité (s). In : Des mots aux dictionnaires : travaux de la section lexicologie, lexicographie, onomastique, toponymie. Actes du XXIIème Congrès international de linguistique et philologie romanes, Bruxelles, Tübingen : Niemeyer. ISBN 978348450373.

DUMAZEDIER, J. - RIPERT, A. 1996. Loisir et culture. Paris : Seuil. ISBN 9782865631766.

GHEBREYESUS, T. A. 2020. Discours d'ouverture du Directeur général de l'OMS lors du point de presse sur COVID-19.

Available online: https://www.who.int/fr/director-general/speeches/detail/whodirector-general-s-opening-remarks-at-the-media-briefing-on-covid-19---11-march2020,

JAMMAL, A. 1992. L'hybridation du langage médical français un phénomène inévitable? In : Journal de radiologie, 73(3), pp. 213-214. ISSN 0221-0363

KOCOUREK, R. 1982. La langue française de la technique et de la science, Vers une linguistique de la langue savante. Wiesbaden: Oscar Brandstetter Verlag. ISBN 9783870971090 .

LE MONDE. Coronavirus et Pandémie de Covid-19.

Available online: https://www.lemonde.fr/coronavirus-2019-ncov

LERAT, P. 1995. Les langues spécialisées. Paris: P.U.F. ISBN 978-2130466024.

PERCEBOIS, J. 2001. Fonctions et vie des sigles et acronymes en contextes de langues anglaise et française de spécialité. In : Meta, vol. 46, n.4, pp. 627-645. ISSN 1492-1421.

XLinguae, Volume 14 Issue 4, October 2021, ISSN 1337-8384, eISSN 2453-711X 
ROCHE, C. 2005. Terminologie et ontologie. In : Langages, vol. 39, n. 157. La terminologie : nature et enjeux. pp. 48-62. Doi : https://doi.org/10.3406/lgge.2005.974 https://www.persee.fr/doc/lgge_0458-726x_2005_num_39_157_974, ISSN 19589549.

ROCHE, C. 2007. Le terme et le concept : fondements d'une ontoterminologie. In : Terminologie \& Ontologie : Théories et Applications Actes de la conférence TOTh, pp. 1-22. ISBN: 2-9516453-7-6.

ROCHE, C. - AMPARO, A. - RUTE, C. 2019. Terminological Resources in the Digital Age. In : Terminology, vol. 25, n. 2, pp. 139-145. DOI: https://doi.org/10.1075/term.00033.roc., consulté le 21 mai 2021. E-ISSN: 1569-9994. ROULEAU, M., 1995. La langue médicale : une langue de spécialité à emprunter le temps d'une traduction. In : TTR : traduction, terminologie, rédaction, vol. $8, \mathrm{n} 2, \mathrm{pp}$. 29-49. ISSN 0835-8443.

VARGAS, E. 2009. Discours de vulgarisation à travers différents médias ou les tribulations des termes scientifiques. In : ILCEA [En ligne], $11 \mid 2009$, mis en ligne le 30 avril 2009, Available online: http://journals.openedition.org/ilcea/217. DOI : https://doi.org/10.4000/ilcea.217. ISBN 978-2-84310-179-3.

VIGNER, G. - MARTIN, A. 1976. Le français technique. Paris : Hachette/Larousse. 978-2010031779

\section{DICTIONNAIRES ET BASES DE DONNEES TERMINOLOGIQUES}

Trésor de la langue française informatisé. Pandémie. Available online: https://www.lalanguefrancaise.com/dictionnaire/definition/pandemie, consulté le 7 mai 2021.

LE PETIT ROBERT, Dictionnaire en ligne, Available online: https://www.lerobert.com/, consulté le 2 mai 2021.

LE PETIT LAROUSSE, Dictionnaire en ligne, Available online: https://www.larousse.fr/, consulté le 23 mai 2021.

TERMIUM Plus, Available online: https://www.btb.termiumplus.gc.ca, consulté le 29 mai 2021.

Words: 5762

Characters: 37671 (20,93 standard pages)

Assoc. Prof. Dr. Ana-Marina Tomescu

Assoc. Prof. Dr. Elena-Cristina Ilinca

University of Piteşti

Department of Applied Foreign Languages

Str. Târgu din Vale, nr. 1

110040, Piteşti Argeş

Romania

marina.tomescu@upit.ro

cristina.ilinca@upit.ro 